Article

\title{
High-Transmittance $2 \pi$ Electrically Tunable Terahertz Phase Shifter with CMOS-Compatible Driving Voltage Enabled by Liquid Crystals
}

\author{
Chan-Shan Yang ${ }^{1,2, *}$, Chun Kuo ${ }^{3}$, Po-Han Chen ${ }^{4}$, Wei-Ta Wu ${ }^{5}$, Ru-Pin Pan ${ }^{5}$, Peichen Yu ${ }^{4}$ and \\ Ci-Ling Pan ${ }^{3, * \mathbb{C}}$ \\ 1 Institute of Electro-Optical Science and Technology, National Taiwan Normal University, \\ Taipei 11677, Taiwan \\ 2 Undergraduate Program of Electro-Optical Engineering, National Taiwan Normal University, \\ Taipei 11677, Taiwan \\ 3 Department of Physics, National Tsing Hua University, Hsinchu 30013, Taiwan; pheobe1212@gmail.com \\ 4 Institute of Electro-Optical Engineering, National Chiao Tung University, Hsinchu 30010, Taiwan; \\ x22761582@hotmail.com (P.-H.C.); yup@faculty.nctu.edu.tw (P.Y.) \\ 5 Department of Electrophysics, National Chiao Tung University, Hsinchu 30010, Taiwan; \\ koala.ep96g@g2.nctu.edu.tw (W.-T.W.); rpchao@gmail.com (R.-P.P.) \\ * Correspondence: csyang@ntnu.edu.tw (C.-S.Y.); clpan@phys.nthu.edu.tw (C.-L.P.); \\ Tel.: +886-2-77346724 (C.-S.Y.); +886-3-5742552 (C.-L.P.)
}

Received: 13 November 2018; Accepted: 8 January 2019; Published: 14 January 2019

\begin{abstract}
We have investigated tunable terahertz $(\mathrm{THz})$ phase shifters that are based on a sandwiched liquid crystal (LC) cell with indium-tin-oxide (ITO) nanowhiskers (NWhs) as transparent electrodes. More than $360^{\circ}$ of phase shift at $1.0 \mathrm{THz}$ was achieved at a driving voltage as low as $\sim 2.6 \mathrm{~V}$ (rms). This is approximately 40 times smaller than that reported in previous works using an electrically tuned LC device. Significance of the NWhs in reducing the required voltage is demonstrated. Overall transmittance of the device is as high as 30\%, which is accountable by absorption losses of ITO NWhs, quartz substrate and LC. Experimental results are in good agreement with a theoretical formulism while taking into account super-thick LC cells $(\sim 1 \mathrm{~mm})$ and pretilt angles. We also propose and demonstrate a novel $\mathrm{THz}$ technique for measuring pretilt angles of liquid crystals.
\end{abstract}

Keywords: far infrared; nanomaterials; terahertz; transparent conductive coatings; phase shift; liquid crystals; birefringence; antireflection coatings

\section{Introduction}

To meet the demands of applications in emerging technologies, such as sub-terahertz (THz) radio-over-fiber communication link [1], numerous quasi-optic components, such as phase shifters [2-10], polarization converter [11-13], filters [14], phase gratings [15], and polarizers [16], have been reported. In particular, a number of tunable $\mathrm{THz}$ devices using liquid crystals (LCs) have received attention [2-17]. For example, an LC spatial THz modulator that can tune THz wavefronts is pivotal in the demonstration of a THz vortex system that can execute orbital angular momentum conversions [17].

Among LC THz devices, magnetically tunable $2 \pi$ phase shifters have been shown to work effectively [2]. They are, however, somewhat bulky and are not so easy to use in some applications. Electrically tunable phase shifters are, therefore, attractive. However, early designs require a high driving voltage (larger than $100 \mathrm{~V}$ ), because they use widely separated lateral electrodes for the lack of electrodes that are transparent in the $\mathrm{THz}$ band [3]. 
Graphene exhibit outstanding electrical conductivity and high transparency in the $\mathrm{THz}$ frequency range $[4,8,10]$. Several groups have demonstrated $\mathrm{THz}$ phase controllers with graphene electrodes $[4,8,10]$. $\mathrm{Y}$. Wu and et al. achieved the phase shift, which is 10.8 degree with a $50 \mu \mathrm{m}$ LC cell [8]. By applying the randomly aligned LCs cell with graphene electrodes T. Sasaki and et al. demonstrated a phase shift of $0.11 \mathrm{rad}$ at $1.5 \mathrm{THz}$ [10]. A phase shift of more than 90 degrees, which is approximately ten times better than that reported in previous designs using LCs and graphene electrodes, was accomplished by our group [4].

Besides, graphene, poly(3,4-ethylenedioxythiophene)/poly(styrenesulfonate) (PEDOT/PSS) films [11], and subwavelength metallic gratings [12] have been employed by Sasaki et al. in electrically tunable $\mathrm{THz}$ polarization converters based on twisted nematic (TN) LC cells as transparent electrodes. The three main advantages are low operating voltages, high transmittance, and simple structures.

Previously, we have shown that indium-tin-oxide (ITO) nanomaterials exhibit outstanding transmittance in the $\mathrm{THz}$ frequencies [18-20]. Their conductivity and DC mobility are also comparable to the sputtered ITO films [18-20]. Later, we also demonstrated the quarter-wave operation or a phase shift of around $\pi / 2$ at $1.0 \mathrm{THz}$ with ITO nanomaterials as transparent electrodes [5]. Interestingly, ITO nanowhiskers have the additional functionality of aligning liquid crystal molecules [5].

Due to the long wavelength of THz waves, thick LC cells are unavoidable. Lest the devices become too unwieldy, a sandwiched LC cell structure for THz application was proposed and demonstrated by us in magnetically tuned phase shifters [2] and patented [21]. With a sandwiched LC cell structure, it is possible to achieve a phase shift of $2 \pi$ at $1.0 \mathrm{THz}$ with a relatively low voltage. Preliminary results were reported by us in a conference paper [22].

In this work, we present in more detail the experimental and theoretical aspects of the device [22]. The frequency-and voltage-dependent characteristics of the $\mathrm{THz}$ phase shifters are described. In particular, a theoretical formulism that can handle thick (approximately $1 \mathrm{~mm}$ ) LC cells is presented. Pretilt angles, which were measured by novel THz techniques, were taken into account. Experimental data are in good agreement with this theoretical formulism.

\section{Experimental Methods}

For use as electrodes, ITO NWhs were deposited on fused silica substrates by using a glancing-angle-incidence e-beam deposition apparatus, as described previously [18]. A photoconductive antenna-based THz time-domain spectrometer (THz-TDS) was employed for characterizing the devices at frequencies of between 0.2 and $1.4 \mathrm{THz}$ [19]. The structural, electrical, and $\mathrm{THz}$ optical properties of ITO nanowhiskers were summarized previously [20]. Briefly, ITO NWhs exhibit superb transparency $(\sim 82 \%)$ in the THz frequency band. Meanwhile, their DC mobility $\left(\sim 92 \mathrm{~cm}^{2} \mathrm{~V}^{-1} \mathrm{~s}^{-1}\right)$ and conductivities $\left(\sim 245 \Omega^{-1} \mathrm{~cm}^{-1}\right)$ are comparable to sputtered ITO thin film.

We studied three different designs of THz phase shifters: types A, B, and C (See Figure 1a). Type A comprised of two identical cells (four substrates) with four ITO NWhs layers; type B consisted of a single cell (three substrates) with three ITO NWhs layers; and, type $C$ was made of a single cell (three substrates) with two ITO NWhs layers. These shifters were constructed by sandwiching the LC (MDA-00-3461 by Merck) layer between fused silica substrates that were deposited with ITO NWhs and then spin-coated by a polyimide (PI) layer, which was rubbed for LC alignment. The substrate thickness was approximately $1.033 \mathrm{~mm}$, whereas that of the LC layer was $\sim 1.12 \mathrm{~mm}$. In other words, the total thickness of the LC material for each design was approximately $2.24 \mathrm{~mm}$. The cells were always biased with sinusoidal signals at $1 \mathrm{kHz}$.

The cross-sectional images by scanning electron microcopy of the substrate deposited with ITO NWhs only, ITO NWhs coated with PI, and further treated with the rubbing process are shown in Figure $1 b-d$, respectively. In Figure 1c, it can be seen that ITO NWhs that were clustered with adjacent whiskers after coating by PI. Besides, the average height of ITO NWhs decreased from $918 \mathrm{~nm}$ to $729 \mathrm{~nm}$ after the rubbing process. The trunks of the ITO NWhs were inclined toward the rubbing 
direction. Therefore, LC molecules in the proximity of the ITO NWhs and the alignment layer will exhibit pretilt angles. Pretilting is thus enhanced by the glancing-angle structure of the ITO NWhs.

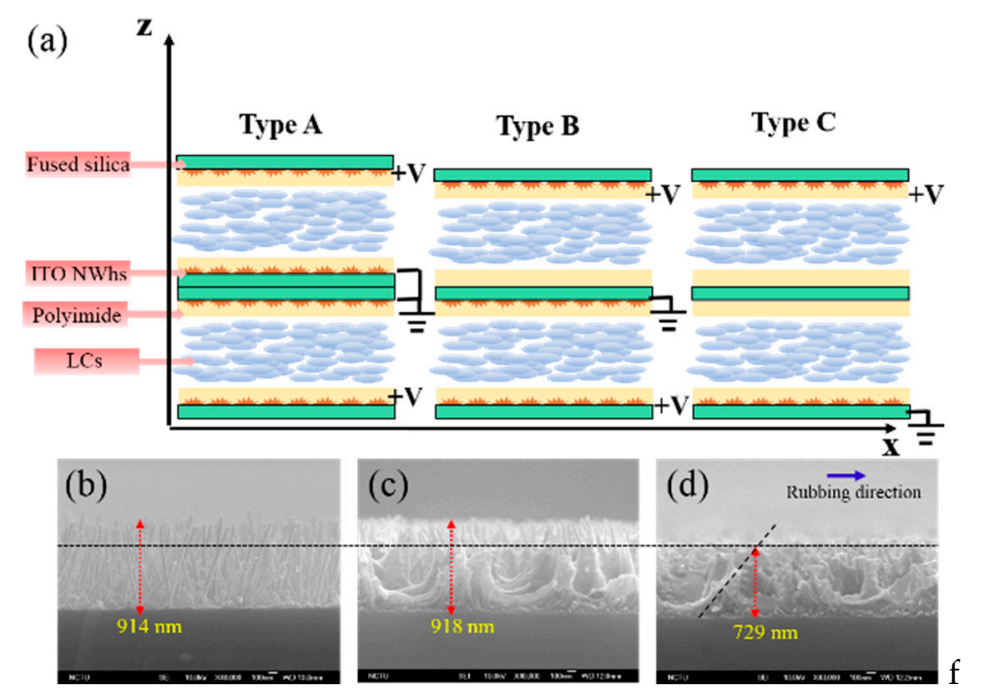

Figure 1. (a) Schematic diagrams of three different configurations of indium-tin-oxide-nanowhiskers (ITO-NWhs)-based phase shifters, type A, B, and C, respectively. The cross-sectional images of (b) the substrate of ITO NWhs, (c) ITO NWhs coated with polyimide, and (d) ITO NWhs treated with the rubbing process.

\section{Theoretical Formulism}

Expected performances of the phase shifters can be analyzed using the approach that we reported earlier [3]. In the absence of the external field, the LC molecules were initially in equilibrium and were oriented more or less parallel to the substrates (Figure 1a). Because of the Fréedericksz transition, when the applied bias was greater than the threshold voltage, the LC molecules will reorient themselves toward the direction of the external field. The threshold voltage for reorienting the LC molecules is given by $V_{\text {th }}=\pi \cdot\left(k_{1} /\left(\varepsilon_{0} \cdot \Delta \varepsilon\right)\right)^{1 / 2} L / d$ [23], where $\varepsilon_{0}=8.854 \times 10^{-12} \mathrm{~F} \cdot \mathrm{m}^{-1}, \Delta \varepsilon=\varepsilon_{/ /}-\varepsilon_{\perp}=11.2$, $k_{1}=12.6 \times 10^{-12} \mathrm{~N}$, and $L$ and $d$ are the free-space permittivity, dielectric anisotropy, splay elastic constants, distance between two electrodes, and thickness of the LC layers, respectively. The maximum tilt angle of the LC director, $\theta_{\max }$, is assumed to be found at the mid-point position of the LC cell, i.e., $\mathrm{z}=d / 2$. Here, the THz wave is assumed to be propagating along the $\mathrm{z}$-direction, normal to the LC cell. The relationship between $\theta_{\max }$ and $V$ can be determined by solving [23]:

$$
\begin{gathered}
\int_{0}^{\frac{\pi}{2}} \frac{\sqrt{\left(1+\zeta \sin ^{2} \theta_{\max } \sin ^{2} \delta\right)\left(1+\rho \sin ^{2} \theta_{\max } \sin ^{2} \delta\right)}}{\sqrt{\left(1-\sin ^{2} \theta_{\max } \sin ^{2} \delta\right)\left(1+\rho \sin ^{2} \theta_{\max } \sin ^{2} \delta\right)}} d \delta \\
=\frac{\pi}{2} \frac{V}{V_{t h}},
\end{gathered}
$$

where $\theta$ is the tilt angle of the LC molecule, $\sin \delta=\sin \theta / \sin \theta_{\max }$. We define $\eta=\sin \theta_{\max }$, then $\sin \theta(z)=$ $\sin \theta_{\max } \cdot \sin \delta^{\prime}(z), \delta^{\prime}(z)$ being the derivative of the integrating variable. In Equation (1), $\zeta=\left(k_{3}-k_{1}\right) / k_{1}$ $\approx 0.22, k_{3}=15.4 \times 10^{-12} \mathrm{~N}, \rho=\left(\varepsilon_{/ /}-\varepsilon_{\perp}\right) / \varepsilon_{\perp} \approx 2.55, \varepsilon_{\perp}=4.4$ for the LC that we used. $V$ is the applied voltage. Equation (1) can be rewritten and used to find $\theta$ at every $z$ position in the LC cell, as follows:

$$
\int_{0}^{\delta^{\prime}} \frac{\sqrt{\left(1+\zeta \eta^{2} \sin ^{2} \delta\right)\left(1+\rho \eta^{2} \sin ^{2} \delta\right)}}{\sqrt{\left(1-\eta^{2} \sin ^{2} \delta\right)\left(1+\rho \eta^{2} \sin ^{2} \delta\right)}} d \delta=\frac{\pi}{2} \frac{E \cdot z}{V_{\mathrm{th}}},
$$

where $E$ is the electric field applied to the device. When considering the effective birefringence of the $\mathrm{LC}$, the total phase shift experienced by the $\mathrm{THz}$ wave propagating through the device, $\Delta \phi$, is given by 


$$
\Delta \phi(V)=\int_{0}^{d} \frac{2 \pi f}{c_{0}}\left[\left(\frac{\cos ^{2} \theta}{n_{\mathrm{o}}^{2}}+\frac{\sin ^{2} \theta}{n_{\mathrm{e}}^{2}}\right)^{-1 / 2}-n_{0}\right] d z,
$$

where $f$ and $c_{0}$ are the frequency and speed, respectively, of the THz wave in vacuum. The extraordinary and ordinary refractive indices of MDA-00-3461 in a frequency range of $0.3-1.4 \mathrm{THz}$ at $25^{\circ} \mathrm{C}$ are essentially frequency-independent: $n_{\mathrm{e}}=1.74$ and $n_{\mathrm{o}}=1.54$, respectively [24]. The aforementioned model is suitable for a thin LC cell (thickness of approximately $20 \mu \mathrm{m}$ or less). However, in this study, the thickness of the LC layer for the $2 \pi \mathrm{THz}$ phase shifter was approximately $1.12 \mathrm{~mm}$, which was approximately 100 times thicker than typical LC devices that were designed in the visible frequency range. In such thick cells, LC molecules more than a few tens of microns away from the cell walls will not be exactly parallel to the rubbed direction in the absence of the applied electric field. Besides, almost all the LC molecules can be easily reoriented in the direction, which is parallel to the external field when the LC cell is biased.

In Figure 2a, we drew a thick LC cell in the e-ray mode without bias. The LC molecules that are sufficiently away from the substrates, highlighted in yellow, are not oriented toward the rubbed direction. After applying a sufficiently high voltage, e.g., $V_{\max }=70 \mathrm{~V}$, we can achieve the o-ray mode for the cell shown in Figure 2b. From the measured phase difference for $\mathrm{THz}$ wave propagation through LC cells in these two modes, we find the maximum possible difference in relative phase retardation, $\Delta \phi\left(V_{\max }\right)$ can be significantly different from the total relative phase shift in the ideal case, $2 \pi f\left(n_{\mathrm{e}}-n_{\mathrm{o}}\right) d / c_{0}$. We propose, therefore, to apply a correction factor for the phase shift,

$$
\alpha=\left[2 \pi f\left(n_{\mathrm{e}}-n_{\mathrm{o}}\right) d / c_{0}\right] / \Delta \phi\left(V_{\max }\right)
$$

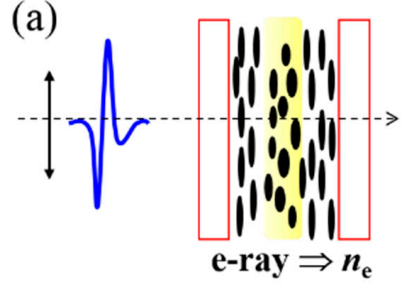

(c)

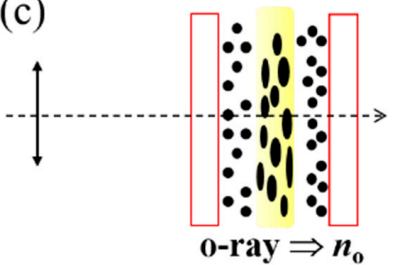

(b)

(d)

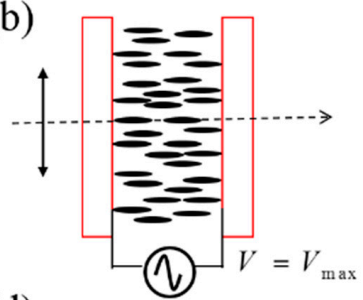

(d)

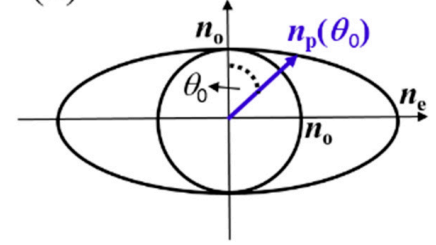

Figure 2. Configurations for determining the correction factors of the thickness $(\alpha)$, and pretilt angle $(\beta)$ of liquid crystal (LC) devices. The cell (a) in the e-ray mode without external field, (b) in the o-ray mode with high driving voltage, and (c) in the o-ray mode without external field. (d) Index ellipsoid used to describe the pretilt angle $\left(\theta_{0}\right)$.

If the LC molecules in the middle of the cell are not perfectly aligned with the rubbing direction because of the weak anchoring ability of the thick LC device, $\alpha>1$.

As mentioned above, the effect of pretilt angle on phase shift in our devices cannot be neglected due to the glancing-angle structure of ITO NWhs. Figure $2 b-d$ are used to describe the experimental method for measuring the pretilt angle of our devices.

We first measured the effective refractive index, $n_{\mathrm{p}}$, which is the sum of the refractive indices in the o-ray mode, $n_{\mathrm{o}}$, Exp, and the birefringence that is caused by the pretilt angle, $\Delta n_{\mathrm{E}}$, in the absence of the external field (see Figure 2c). The same procedure was repeated with the cell biased at $70 \mathrm{~V}(\mathrm{rms})$, as shown in Figure $2 \mathrm{~b}$. The parameters, $\Delta \phi_{\mathrm{E}}$, and $n_{\mathrm{p}}$ are then related by 


$$
n_{\mathrm{p}}\left(\theta_{0}\right)=\Delta n_{\mathrm{E}}+n_{\mathrm{o}, \operatorname{Exp}}=\frac{c_{0}}{2 \pi f d} \Delta \phi_{\mathrm{E}}+n_{\mathrm{o}, \operatorname{Exp}}
$$

where $\theta_{0}$ is the pretilt angle. On the other hand, $n_{\mathbf{p}}$ can also be determined by using the index ellipsoid shown in Figure 2d,

$$
n_{\mathrm{p}}\left(\theta_{0}\right)=\left[\frac{\sin ^{2}\left(\theta_{0}\right)}{n_{\mathrm{e}}^{2}}+\frac{\cos ^{2}\left(\theta_{0}\right)}{n_{\mathrm{o}}^{2}}\right]^{-1 / 2} .
$$

The pretilt angle can then be determined by the above procedure and applying Equations (5) and (6). Taking into account of the thick cell and effect of the pretilt angle mentioned above, we obtain an expression for the effective phase shift,

$$
\Delta \phi_{\text {eff }}=(\alpha)(\beta)(\Delta \phi),
$$

where the compensation factor for pretilt, $\beta$, is given by,

$$
\beta=c_{0} d \Delta \phi_{\mathrm{E}}\left(V_{\max }\right) /(2 \pi f) /\left(n_{\mathrm{e}}-n_{\mathrm{o}}\right) .
$$

For the case of $\beta<1$, the LC cell exhibits a pretilt angle in the absence of the bias electric field.

\section{Results and Discussions}

Figure 3 shows plots of the experimentally measured transmittances of the three types of phase shifters. The cumulative transmittances for the THz phase shifters of type A, B, and C, reduced due to absorption losses of fused silica, LCs, and ITO NWhs were $20 \%, 25 \%$, and $31 \%$, respectively. The difference is mainly due to the numbers of substrates and ITO NWhs layers that were employed in the three configurations.

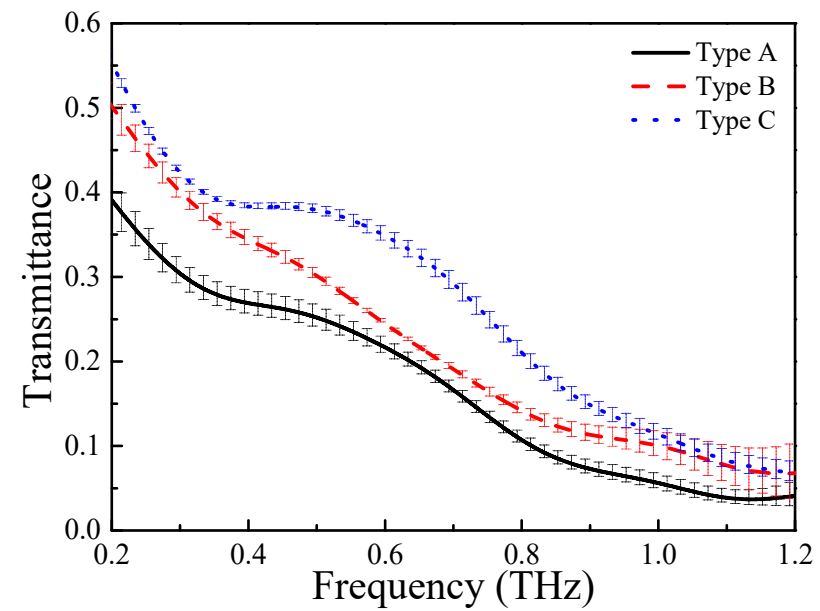

Figure 3. Tunable terahertz (THz) transmittance of the phase shifters, type A, B, and C, respectively.

The transmittance of $\pi / 2$ phase shifters using ITO NWhs as electrodes ( $\geq 75 \%)$ is much more superior than that using sputtered ITO film $(\leq 1 \%)$ [5]. Therefore, in this case of $2 \pi$ waveplate with multi-cell structure using ITO NWhs as electrodes, transmittance of $20 \sim 30 \%$ is reasonable and much superior to the previous state-of-art result. Using complex permittivities or refractive indices of ITO NWhs, fused Silica, and LCs, reported in our previous works [18-20,24] in a Jones matrix formulation with Fresnel equations, we can estimate the loss caused from different interfaces of the device. The analysis shows that the most serious sources of loss for our device come from the interfaces of fused Silica/ITO NWhs, and LCs/ITO NWhs. On the other hand, losses incurred at air/ fused Silica and LCs / fused Silica interfaces are slight. 
In Figure 4, we have plotted phase shift as a function of frequency from 0.2 to $1.0 \mathrm{THz}$ by varying the voltages for the three types of devices. As expected, the measured phase shift changed linearly with frequency for a given bias voltage. For the type-A phase shifter, the slope of linear fits varied from $0.40 \pi / \mathrm{THz}$ to $2.67 \pi / \mathrm{THz}$ as the applied voltage was ramped from 2.12 to $10.61 \mathrm{~V}$ (rms). For the type-B and type-C structures, they varied from $0.51 \pi / \mathrm{THz}$ to $2.49 \pi / \mathrm{THz}$, and $0.60 \pi / \mathrm{THz}$ to $2.59 \pi / \mathrm{THz}$, as the applied voltages was ramped from 2.40 to $10.61 \mathrm{~V}$ (rms) and 5.66 to $21.21 \mathrm{~V}$ (rms), respectively.
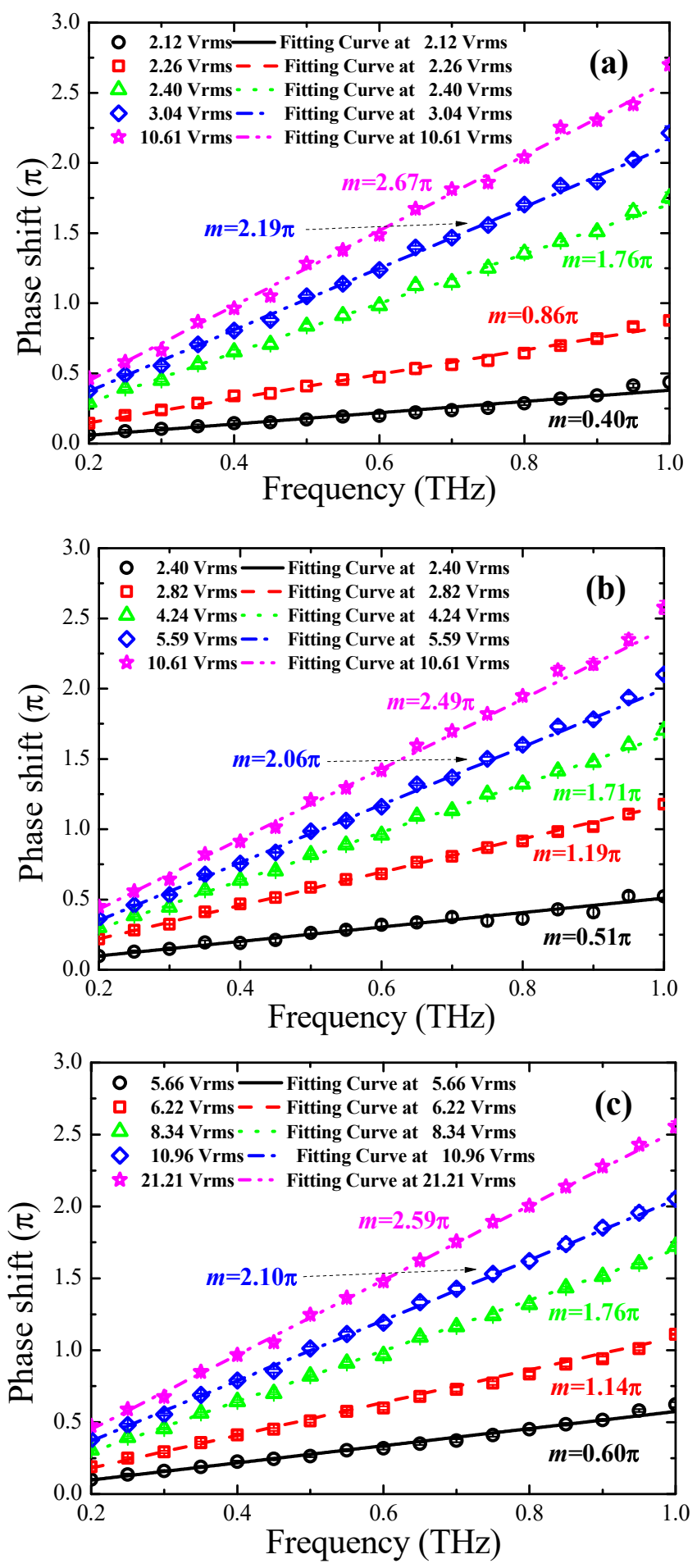

Figure 4. $\mathrm{THz}$ Phase shift as a function of $\mathrm{THz}$ frequency for five driving voltages, respectively, for devices of (a) type A, (b) type B, and (c) type C configurations. 
In Figure 5, we have plotted experimentally measured phase shifts as a function of the driving voltage. The theoretical curve according to the model proposed in this study and those use earlier ones that are based on simpler models with approximations. References $[3,8]$ are also shown.
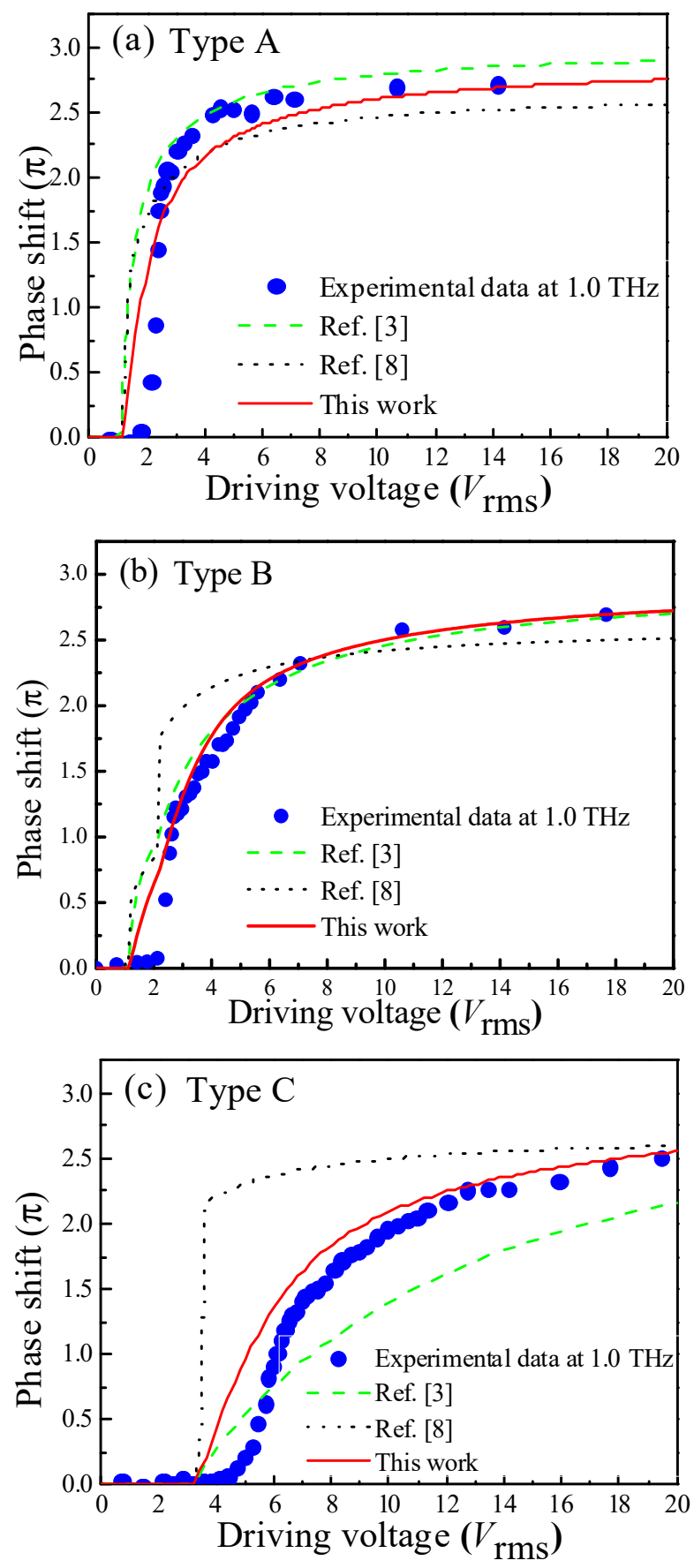

Figure 5. Phase shift as a function of applied voltage for devices of (a) type A, (b) type B, and (c) type C configurations.

In all cases, more than $360^{\circ}$ degrees of phase shifts was achieved for all three types of devices. Further, Bias voltages for achieving $2 \pi$ phase shift at $1.0 \mathrm{THz}$ were $\mathrm{V}(\mathrm{rms}) \approx 2.6,5.3,10.3$ for types $\mathrm{A}, \mathrm{B}$, and $C$ devices, respectively. Thus, the effect of ITO NWhs for assisting the alignment and reorientation of LC molecules is significant. Further, this characteristic for type A device is compatible with CMOS technology. We measured the response time properties of the present ITO NWhs phase shifter. The 20 to $80 \%$ rise time and fall time are around $52 \mathrm{~s}$ and $240 \mathrm{~s}$, respectively. 
Overall, the agreement of experimental data with the present model is significantly better than previous ones. To check quantitatively the accuracy of the theoretical models, we define an error function, $\Delta=\Sigma\left|\Lambda_{\mathrm{j}, \text { theo }}-\Lambda_{\mathrm{j}, \exp }\right|$, where $j$ equals $1,2,3, \ldots$ The parameters, $\Lambda_{\mathrm{j}, \exp }$ and $\Lambda_{\mathrm{j}, \text { theo }}$, represent the experimental and theoretical phase shift when a bias voltage, $V_{\mathrm{j}}$, was applied. For the case of data shown in Figure 5a for type A and employing the theoretical model that is described in Refs. $[3,8]$, and this work, $\Delta$ were found to be $8.19,8.38$, and 6.51 , respectively. For the case of data shown in Figure $5 b, c$ for type B and C, similar sets of values for $\Delta$ were found to be $(7.06,16.11,4.25)$, and $(17.23,41.89,10.96)$, respectively. These analyses further confirmed that the theoretical method that is presented in this work is in closer agreement with the experimental data.

Finally, we summarize the experimental data and calculated parameters for the three types of devices in Table 1.

Table 1. Experimentally measured phase shifts, birefringence, pretilt angle $\left(\theta_{0}\right)$, operating voltage, compensation factor of thick thickness $(\alpha)$, pretilt angles $(\beta)$, and average transmittance for the $2 \pi$ phase shift of the THz phase shifter for the types A, B, and C configurations, separately.

\begin{tabular}{cccc}
\hline & Type A & Type B & Type C \\
\hline$\Delta \phi_{\mathrm{E}}$ at $1 \mathrm{THz}$ & $2.750 \pi$ & $2.832 \pi$ & $2.690 \pi$ \\
$\Delta n_{\mathrm{E}}$ & 0.183 & 0.191 & 0.177 \\
$\theta_{0}$ & $19.07^{\circ}$ & $15.98^{\circ}$ & $21.42^{\circ}$ \\
$\alpha$ & 1.064 & 1.081 & 1.187 \\
$\beta$ & 0.915 & 0.955 & 0.885 \\
Bias voltage for $2 \pi$ phase shift at $1.0 \mathrm{THz} \mathrm{V}(\mathrm{rms})$ & 2.6 & 5.3 & 10.3 \\
Averaged transmittance & $20.0 \%$ & $25.4 \%$ & $31.3 \%$ \\
\hline
\end{tabular}

\section{Conclusions}

In this work, we theoretically and experimentally studied tunable terahertz (THz) phase shifters that are based on a sandwiched liquid crystal (LC) cell with ITO nanowhiskers (NWhs) as transparent electrodes. Three designs were presented. All achieved more than $360^{\circ}$ of phase shift at $1.0 \mathrm{THz}$. The bias required was as low as $\sim 2.6 \mathrm{~V}$ (rms), while the highest throughput was more than $30 \%$ for Type A devices. The bias voltage reported is a new record, which is approximately 40 times smaller than that reported previously using electrically tunable LC device. Such operating characteristics are compatible with CMOS technology. Significance of the NWhs in reducing the required voltage is demonstrated. The experimental results are in good agreement with a theoretical formulism that took into account super-thick LC cells $(\sim 1 \mathrm{~mm})$ and effect of the pretilt. We also demonstrated a new method for measuring pretilt angles of LC cells by a novel THz technique.

Author Contributions: C.-S.Y., C.K., and C.-L.P. conceived the research. C.-S.Y. and C.K. carried out optical measurements, analysed the data, and performed theoretical analysis. C.-S.Y. fabricated the devices and received assistance from C.K., P.-H.C., W.-T.W., R.-P.P., and P.Y., C.-S.Y. and C.-L.P. wrote the manuscript with inputs from all authors.

Funding: This study was partially funded by grant 104-2221-E-007-093-MY3 and others of the Ministry of Science Technology, as well as the Academic Top University Program of the Ministry of Education, Taiwan.

Acknowledgments: C.-L.P. would like to thank the U.S. Air Force Office of Scientific Research for support, AOARD FA2386-13-1-4086.

Conflicts of Interest: The authors declare no conflicts of interest.

\section{References}

1. Shi, J.-W.; Huang, C.-B.; Pan, C.-L. Millimeter-wave photonic wireless links for very-high data rate communication. NPG Asia Mater. 2011, 3, 41-48. [CrossRef]

2. Chen, C.-Y.; Hsieh, C.-F.; Lin, Y.-F.; Pan, R.-P.; Pan, C.-L. Magnetically tunable room-temperature $2 \pi$ liquid crystal terahertz phase shifter. Opt. Express 2004, 12, 2630-2635. [CrossRef] 
3. Wu, H.-Y.; Hsieh, C.-F.; Tang, T.-T.; Pan, R.-P.; Pan, C.-L. Electrically tunable room-temperature $2 \pi$ liquid crystal terahertz phase shifter. IEEE Photonic Technol. Lett. 2006, 18, 1488-1490.

4. Yang, C.-S.; Kuo, C.; Tang, C.-C.; Chen, J.C.; Pan, R.-P.; Pan, C.-L. Liquid-crystal terahertz quarter-wave plate using chemical-vapor-deposited graphene electrodes. IEEE Photonics J. 2015, 7, 2200808. [CrossRef]

5. Yang, C.-S.; Tang, T.-T.; Pan, R.-P.; Yu, P.; Pan, C.-L. Liquid crystal terahertz phase shifters with functional indium-tin-oxide nanostructure for biasing and alignment. Appl. Phys. Lett. 2014, 104, 141106. [CrossRef]

6. Lin, X.-W.; Wu, J.-B.; Hu, W.; Zheng, Z.-G.; Wu, Z.-J.; Zhu, G.; Xu, F.; Jin, B.-B.; Lu, Y.-Q. Self-polarizing terahertz liquid crystal phase shifter. AIP Adv. 2011, 1, 032133. [CrossRef]

7. Altmann, K.; Reuter, M.; Garbat, K.; Koch, M.; Dabrowski, R.; Dierking, I. Polymer stabilized liquid crystal phase shifter for terahertz waves. Opt. Express 2013, 21, 12395-12400. [CrossRef] [PubMed]

8. Wu, Y.; Ruan, X.; Chen, C.-H.; Shin, Y.J.; Lee, Y.; Niu, J.; Liu, J.; Chen, Y.; Yang, K.-L.; Zhang, X.; et al. Graphene/liquid crystal based terahertz phase shifters. Opt. Express 2013, 21, 21395-21402. [CrossRef] [PubMed]

9. Du, Y.; Tian, H.; Cui, X.; Wang, H.; Zhou, Z.-X. Electrically tunable liquid crystal terahertz phase shifter driven by transparent polymer electrodes. J. Mater. Chem. C 2016, 4, 4138-4142. [CrossRef]

10. Sasaki, T.; Noda, K.; Kawatsuki, N.; Ono, H. Universal polarization terahertz phase controllers using randomly aligned liquid crystal cells with graphene electrodes. Opt. Lett. 2015, 40, 1544-1547. [CrossRef] [PubMed]

11. Sasaki, T.; Okuyama, H.; Sakamoto, M.; Noda, K.; Okamoto, H.; Kawatsuki, N.; Ono, H. Twisted nematic liquid crystal cells with rubbed poly(3,4-ethylenedioxythiophene)/poly(styrenesulfonate) films for active polarization control of terahertz waves. J. Appl. Phys. 2017, 121, 143106. [CrossRef]

12. Sasaki, T.; Kushida, H.; Sakamoto, M.; Noda, K.; Okamoto, H.; Kawatsuki, N.; Ono, H. Liquid crystal cells with subwavelength metallic gratings for transmissive terahertz elements with electrical tunability. Opt. Commun. 2019, 431, 63-67. [CrossRef]

13. Vasić, B.; Zografopoulos, D.C.; Isić, G.; Beccherelli, R.; Gajić, R. Electrically tunable terahertz polarization converter based on overcoupled metal-isolator-metal metamaterials infiltrated with liquid crystals. Nanotechnology 2017, 28, 124002. [CrossRef] [PubMed]

14. Chen, C.-Y.; Pan, C.-L.; Hsieh, C.-F.; Lin, Y.-F.; Pan, R.-P. Liquid-crystal-based terahertz tunable Lyot filter. Appl. Phys. Lett. 2006, 88, 101107. [CrossRef]

15. Lin, C.-J.; Li, Y.-T.; Hsieh, C.-F.; Pan, R.-P.; Pan, C.-L. Manipulating terahertz wave by a magnetically tunable liquid crystal phase grating. Opt. Express 2008, 16, 2995-3001. [CrossRef] [PubMed]

16. Hsieh, C.-F.; Lai, Y.-C.; Pan, R.-P.; Pan, C.-L. Polarizing terahertz waves with nematic liquid crystals. Opt. Lett. 2008, 33, 1174-1176. [CrossRef] [PubMed]

17. Xie, Z.; Wang, X.; Ye, J.; Feng, S.; Sun, W.; Akalin, T.; Zhang, Y. Spatial terahertz modulator. Sci. Rep. 2013, 3, 3347. [CrossRef]

18. Yang, C.-S.; Chang, C.-M.; Chen, P.-H.; Yu, P.; Pan, C.-L. Broadband terahertz conductivity and optical transmission of indium-tin-oxide (ITO) nanomaterials. Opt. Express 2013, 21, 16670-16682. [CrossRef] [PubMed]

19. Yang, C.-S.; Lin, M.-H.; Chang, C.-H.; Yu, P.; Shieh, J.-M.; Shen, C.-H.; Wada, O.; Pan, C.-L. Non-Drude behavior in indium-tin-oxide nanowhiskers and thin films investigated by transmission and reflection $\mathrm{THz}$ time-domain spectroscopy. IEEE J. Quantum Electron. 2013, 49, 677-690. [CrossRef]

20. Pan, C.-L.; Yang, C.-S.; Pan, R.-P.; Yu, P.; Lin, G.-R. Nanostructured Indium Tin Oxides and other Transparent Conducting Oxides: Characteristics and Applications in the THz Frequency Range. In Terahertz Spectroscopy-A Cutting Edge Technology; Chapter 14; Uddin, J., Ed.; InTech Open: London, UK, 2017; pp. 267-286.

21. Pan, C.-L.; Chao, R.-P.; Chen, C.-Y. Tunable THz Phase Shifters by Magnetic-Field-Controlled Birefringence in Liquid Crystals. Taiwan (ROC) Patent 200186, 11 April 2004.

22. Pan, C.-L.; Pan, R.-P.; Yang, C.-S.; Yu, P. Voltage-controlled $2 \pi$ liquid-crystal terahertz phase shifter with indium-tin-oxide (ITO) nanowhiskers as transparent electrodes. In Proceedings of the XXXI General Assembly of the International Union of Radio Science, Beijing, China, 16-13 August 2014. Paper DFC01.6.

23. Yeh, P.; Gu, C. Optics of Liquid Crystal Displays; Wiley: Hoboken, NJ, USA, 1999.

24. Ku, C.-P.; Shih, C.-C.; Lin, C.-J.; Pan, R.-P.; Pan, C.-L. THz Optical Constants of the Liquid Crystal MDA-00-3461. Mol. Cryst. Liquid Cryst. 2011, 541, 303-308. [CrossRef]

(C) 2019 by the authors. Licensee MDPI, Basel, Switzerland. This article is an open access article distributed under the terms and conditions of the Creative Commons Attribution (CC BY) license (http:/ / creativecommons.org/licenses/by/4.0/). 